\title{
Estado nutricional-hematológico y parasitosis intestinal de niños escolares de 5 a 12 años de cuatro localidades rurales de Paraguay
}

\author{
Valentina Díaz, Patricia Funes, Gloria Echagüe, Liliana Sosa, Irene Ruiz, Jorge Zenteno, Lourdes Rivas, \\ Dominich Granado
}

Departamento de Bioquímica Clínica. Instituto de Investigaciones en Ciencias de la Salud, Universidad Nacional de Asunción. Paraguay

Cómo referenciar este artículo/ How to reference this article:
Díaz V, Funes P, Echagüe G, Sosa L, Ruiz I, Zenteno J, Rivas L, Granado D. Estado nutricionalhematológico y parasitosis intestinal de niños escolares de 5 a 12 años de cuatro localidades rurales de Paraguay. Mem. Inst. Investig. Cienc. Salud. 2018; 16(1): 26-32

\section{RE S U M E N}

El mundo, actualmente se enfrenta a una doble carga de malnutrición que incluye la desnutrición y la alimentación excesiva. A ello se suman las parasitosis intestinales que es una enfermedad frecuente con importante morbimortalidad en la población infantil, ligadas a la pobreza y malas condiciones higiénico-sanitarias. El objetivo de este trabajo fue describir el estado nutricional-hematológico y parasitológico de niños escolares de cuatro comunidades rurales de Paraguay. Estudio observacional descriptivo de corte transverso en el que participaron 102 niños de ambos sexos de 5 a 12 años de edad. Se realizó medición de peso y talla, utilizando balanza calibrada, y un altímetro fijado a la pared. Toma de muestra sanguínea por punción venosa para determinación de parámetros hematológicos, procesados en contador hematológico por impedancia. Muestras de heces de una sola toma fueron recogidas en frascos apropiados con formol al 10\%, utilizándose 4 métodos: directo, flotación de Willis, Graham y de concentración. En relación al estado nutricionalhematológico se encontró que el 3,9\% de los niños estaba con desnutrición moderada y el $9,8 \%$ presentó riesgo de desnutrición; anemia se observó en el 38,2\% de los niños. En relación a la parasitosis, el estudio diagnóstico se realizó a 94 niños y se encontró que el $72,2 \%$ estaba parasitado, siendo Blastocystis hominis el más frecuente. Tanto la frecuencia de anemia como de parasitosis es alta en esta población, sin embargo no se pudo establecer una relación entre ellas.

Palabras clave: anemia, desnutrición, parasitosis, niños escolares.

\section{A B S T R A C T}

The world is currently facing a double burden of malnutrition that includes malnutrition and over-feeding. In addition, intestinal parasitoses are a frequent disease with significant morbidity and mortality in children, related to poverty and poor hygienic-sanitary conditions. The objective of this study was to evaluate the nutritional-hematological and parasitological state of school children from four rural communities of Paraguay. Crosssectional observational descriptive study that included 102 children of both sexes, 5 to 12 years of age. Measurement of weight, height, blood sample and stool collection were performed. Regarding the nutritional-hematological state, $3.9 \%$ of the children were moderately undernourished, $9.8 \%$ were at risk of malnutrition and $38.2 \%$ of the children had anemia. In relation to parasitosis, the diagnostic study was performed in 94 children and it was found that $72.2 \%$ was parasitized, and Blastocystis hominis was the most frequent. A high frequency of anemia and parasitosis was found in this population; however no relationship was established between them.

Keywords: anemia, malnutrition, parasitosis, school children. 


\section{INTRODUCCIÓN}

La nutrición es la ingesta de alimentos en relación con las necesidades dietéticas del organismo. Una buena nutrición (una dieta suficiente y equilibrada combinada con el ejercicio físico regular) es un elemento fundamental de la buena salud. Una mala nutrición puede reducir la inmunidad, aumentar la vulnerabilidad a las enfermedades, alterar el desarrollo físico y mental, y reducir la productividad. En la actualidad, el mundo se enfrenta a una doble carga de malnutrición que incluye la desnutrición y la alimentación excesiva. La malnutrición también se caracteriza por la carencia de diversos nutrientes esenciales en la dieta, en particular hierro, ácido fólico, vitamina A y yodo. La desnutrición contribuye a cerca de un tercio de todas las muertes infantiles ${ }^{(1,2)}$.

El estado nutricional se encuentra relacionado con las condiciones de pobreza de una población, es decir que, aquellas personas que se encuentran en pobreza y especialmente en pobreza extrema, tienen mayores riesgos de tener un consumo escaso de nutrientes necesarios para un desarrollo físico e intelectual normal ${ }^{(3)}$. En un trabajo realizado en una población pediátrica de una comunidad rural de Yvyraty (Paraguarí -Paraguay) se encontró que el 70,2 \% de los niños era normopeso, 14,9\% estaba en riesgo de desnutrición, 6,4 por ciento con desnutrición moderada y $8,5 \%$ con sobrepeso y el $19,1 \%$ presentaron anemia ${ }^{(4)}$.

La base de datos mundial de la OMS sobre la anemia es la única fuente de estimaciones de la anemia a nivel nacional, regional y mundial. Se emplea como indicador la concentración de hemoglobina en sangre ${ }^{(5)}$. La deficiencia de hierro es la deficiencia nutricional más prevalente y la principal causa de anemia a escala mundial. En los países en vías de desarrollo los grupos más afectados son los niños y adolescentes, debido a sus mayores requerimientos determinados por el crecimiento ${ }^{(6)}$. En Paraguay, en un estudio realizado en escolares en zonas aledañas al rio Paraguay, el $11,8 \%$ de esos niños presentaron anemia ${ }^{(7)}$.

Las enfermedades parasitarias constituyen una importante carga de enfermedad en todo el mundo, sobre todo, pero no exclusivamente, en países en vías de desarrollo. Las parasitosis intestinales son una enfermedad frecuente con importante morbimortalidad en la población infantil, ligadas a la pobreza y malas condiciones higiénico-sanitarias ${ }^{(8)}$.

Según datos de la OPS/OMS el 20-30\% de todos los latinoamericanos están infectados por parásitos intestinales transmitidos por contacto con el suelo, estas cifras pueden aumentar hasta el $50 \%$ en los barrios pobres, e inclusive en algunas tribus indígenas llegar al 95\%.Dentro de los objetivos del milenio, la desparasitación ha tenido impacto y se ha demostrado que ésta puede prevenir $82 \%$ del retraso en el crecimiento y es responsable de $35 \%$ del aumento de peso en niños en edad preescolar con malnutrición. Además, reduce el ausentismo escolar en $25 \%$ mejorando la escolarización y los resultados en la salud en general ${ }^{(9)}$.

Un trabajo realizado en Urabá (Colombia) en menores de 15 años encontró una desnutrición de $25 \%$, hemoglobina menor a $11 \mathrm{~g} / \mathrm{dL}$ y una parasitosis intestinal de $87 \%{ }^{(10)}$.

En Paraguay, de cada 1000 niños y niñas que nacen, 19 mueren antes de alcanzar los 5 años, 16 antes de cumplir el primer año y 11 antes del primer mes de vida. Asimismo, el $44,1 \%$ de la niñez menor de 5 años sufre o está en riesgo de desnutrición ${ }^{(11)}$.

En estudios realizados en Paraguay en 1997 se han encontrado altas tasas de parasitosis en niños escolares de zonas aledañas al río Paraguay en Asunción ${ }^{(12)}$, como también en Ciudad del Este en el $2015^{(13)}$, y en el 2016 en niños de comunidades indígenas de Alto Paraná(14).

Este trabajo tuvo como objetivo evaluar el estado nutricional, hematológico y parasitológico de niños escolares de 5 a 12 años de cuatro localidades rurales de Paraguay.

\section{MATERIALES Y MÉTODOS}

Estudio de diseño observacional descriptivo de corte transverso, con muestreo no probabilístico por conveniencia. De los 148 niños de ambos sexos que fueron convocados, 102 reunieron los criterios de inclusión. Participaron escolares de 5 a 12 de edad que asistieron a las escuelas rurales de "San Felipe" de Arroyos y Esteros, "Juan Gregorio Olmedo de Mbocayaty del Yhaguy, "Loma Conche" de Chaco -í, y "San Antonio" de Puerto Elsa, durante el año 2008. Se solicitó entrevista a las autoridades de las escuelas seleccionadas y se les explicó todo lo relacionado al proyecto. Luego se realizó charlas explicativas a los padres para brindarles información detallada del estudio, y los beneficios 
que obtendrían, los requisitos y condiciones que debían presentar, más la entrega de un consentimiento informado que fue firmado por los mismos. El día del estudio se realizó entrevista a los padres o tutores de los alumnos y luego la medición del peso y talla; seguidamente la extracción de la muestra sanguínea y finalmente la recolección de muestra de heces. Los resultados fueron entregados manteniendo la confidencialidad.

A cada niño se le tomaron medidas antropométricas como el peso y la talla. El peso fue medido por una balanza calibrada de 0 a 120 kilogramos con precisión de 100 miligramos y ajustada a cero antes de cada medición. Y luego la talla con un altímetro graduado en centímetros y décimas de centímetros fijada a la pared. El estado nutricional se evaluó según criterio de la OMS para el indicador IMC/edad, con los siguientes puntos de corte: <3DE: desnutrición grave; <-2DE hasta -3DE: desnutrición moderada; <-1DE hasta $-2 \mathrm{DE}$ : riesgo de desnutrición; entre -1 y +1DE: no tiene desnutrición o adecuado; > +1 DE hasta +2DE: sobrepeso; > 2DE: obesidad ${ }^{(15)}$. Dentro del perfil hematológico se midieron la hemoglobina, el hematocrito y los índices hematimétricos: el volumen corpuscular medio (VCM), la hemoglobina corpuscular media (HCM) y la concentración de la hemoglobina corpuscular media ( $\mathrm{CHCM})$. Para ello se obtuvo muestra sanguínea con punción venosa con jeringas descartables, en un frasco con anticoagulante EDTA (etilendiamino tetra acético $)^{(16,17)}$. Estas muestras fueron procesadas en contador hematológico ABX Micros 60 OT (Francia).

Las muestras de heces fueron recogidas en frascos apropiados con formol al $10 \%$. Para el análisis parasitológico se utilizó el método directo en solución salina fisiológica y coloración con lugol, el método de flotación de Willis y el de concentración éter formalina al 10\%. La búsqueda de Enterobius vermicularis se realizó por el método de Graham ${ }^{(18)}$.

\section{RESULTADOS}

La población estudiada estuvo compuesta por 102 niños de ambos sexos, de los cuales $58(56,9 \%)$ fueron del sexo masculino. La edad de los niños comprendió de 5 a 12 años, siendo la edad media de $9 \pm 2$ años. Los valores medios de peso y talla fueron significativamente más elevados en los niños en relación a las niñas.

Al determinar el estado nutricional según la OMS para el indicador IMC/edad se encontró que $3.9 \%$ de los niños estaban con desnutrición moderada, el 9,8\% presentó riesgo de desnutrición y $65,7 \%$ peso adecuado, en tanto que en el $20,6 \%$ de los niños presento sobrepeso u obesidad. Las características generales diferenciado por sexo se presentan en la Tabla 1.

Tabla 1. Características generales de los niños participantes

\begin{tabular}{|c|c|c|c|c|}
\hline & $\begin{array}{l}\text { Total } \\
(n=102)\end{array}$ & $\begin{array}{l}\text { Femenino } \\
(n=44)\end{array}$ & $\begin{array}{l}\text { Masculino } \\
(n=58)\end{array}$ & $\mathbf{p}$ \\
\hline Edad(años) ${ }^{*}$ & $9 \pm 2$ & $8 \pm 2$ & $9 \pm 2$ & $p<0.05$ \\
\hline Peso $(\mathbf{k g})^{*}$ & $25,9 \pm 6,1$ & $23,9 \pm 25,7$ & $27,4 \pm 6,0$ & $p<0.05$ \\
\hline Talla $(\mathrm{cm})^{*}$ & $124,1 \pm 10,5$ & $120,5 \pm 10,6$ & $126,7 \pm 9,6$ & $p<0.05$ \\
\hline $\operatorname{IMC}\left(\mathrm{kg} / \mathrm{m}^{2}\right)$ & $16,6 \pm 2,2$ & $16,2 \pm 2,1$ & $16,9 \pm 2,3$ & NS \\
\hline $\begin{array}{l}\text { Estado nutricional por el } \\
\text { indicador IMC/sexo }(\%) \\
\text { Desnutrición moderada }\end{array}$ & $4(3,9 \%)$ & $2(4,5 \%)$ & $2(3,4 \%)$ & NS \\
\hline Riesgo de desnutrición & $10(9,8 \%)$ & $5(11,4 \%)$ & $5,0(8,6 \%)$ & \\
\hline Adecuado & $67(65,7 \%)$ & $28(63,6 \%)$ & $39(67,2 \%)$ & \\
\hline Sobrepeso & $17(16,7 \%)$ & $7(15,9 \%)$ & $10(17,2 \%)$ & \\
\hline Obesidad & $4(3,9 \%)$ & $2(4,5 \%)$ & $2(3,4 \%)$ & \\
\hline
\end{tabular}

Los parámetros hematológicos se muestran diferenciados por sexo en la Tabla 2 . Se observó que la media de los valores de hemoglobina fue de $12 \pm 1 \mathrm{~g} / \mathrm{dL}$ sin diferencia significativa según sexo. La frecuencia de anemia encontrada fue de 38,2 \% (39/102) en la población total de niños (Figura 1). 
Díaz $V$ et al. Estado nutricional-hematológico y parasitosis intestinal de niños escolares...

Tabla 2. Parámetros hematológicos de la población estudiada

\begin{tabular}{lcccl}
\hline Parámetros hematológicos & $\begin{array}{c}\text { Total* } \\
(\mathbf{n = 1 0 2})\end{array}$ & $\begin{array}{l}\text { Femenino } \\
(\mathbf{n = 4 4 )}\end{array}$ & $\begin{array}{c}\text { Masculino* } \\
(\mathbf{n = 5 8})\end{array}$ & $\mathbf{P}$ \\
\hline Hemoglobina (g/dL) & $12 \pm 1$ & $12 \pm 1$ & $12 \pm 1$ & $\mathrm{NS}$ \\
Hematocrito (\%) & $38 \pm 5$ & $38 \pm 7$ & $37 \pm 3$ & $\mathrm{NS}$ \\
Índices Hematimétricos & & & & \\
VCM (fL) & $84 \pm 7$ & $83 \pm 9$ & $82 \pm 4$ & $\mathrm{NS}$ \\
HCM (pg) & $30 \pm 3$ & $34 \pm 4$ & $27 \pm 2$ & $\mathrm{NS}$ \\
CHCM (gr/dL) & $32 \pm 3$ & $32 \pm 5$ & $33 \pm 9$ & NS
\end{tabular}

$*$ (Media $\pm \mathrm{DE})$

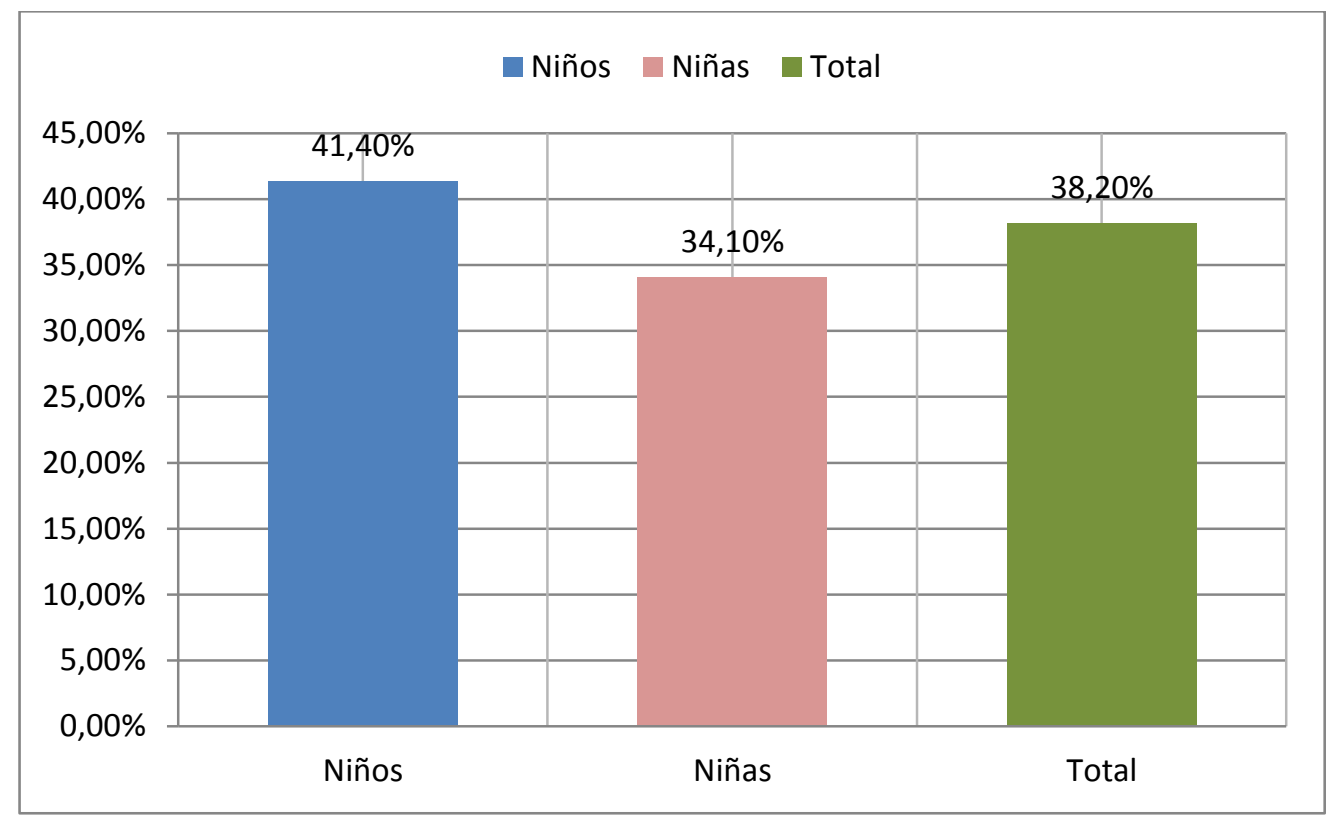

Figura 1. Frecuencia de anemia en la población general y según sexo

De los 102 niños escolares a 94 se les realizó el estudio parasitológico y se encontró que el $72,2 \%$ (68/94) estaba parasitado, con frecuencia similares respecto al sexo. Por el método de Graham se observó Enterobius vermicularis en el 16,2\% (16/68). Los demás parásitos intestinales hallados por los métodos directo y de concentración, se detallan en la Figura 2.

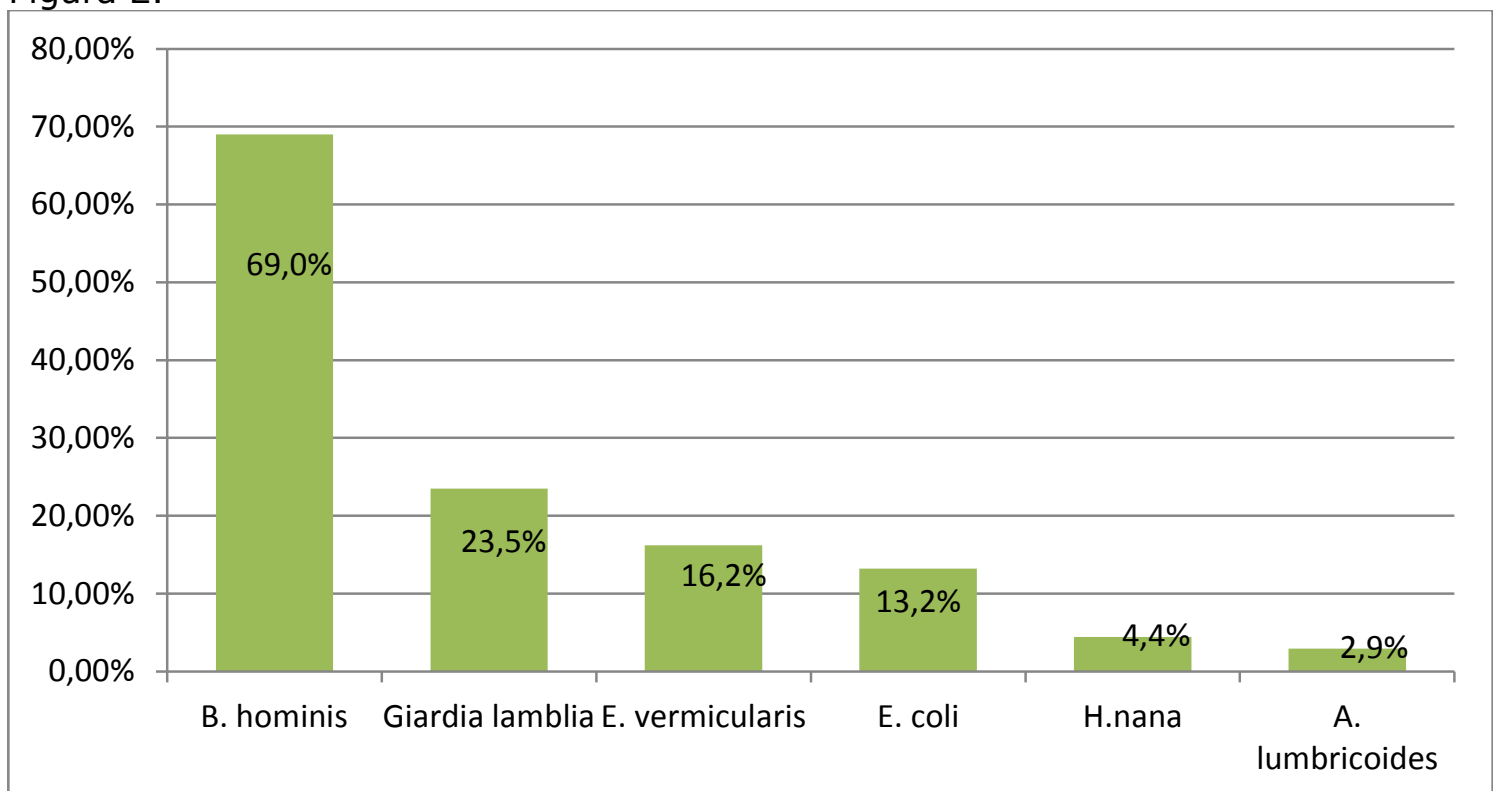

Figura 2. Frecuencia de tipos de parasitosis encontrada en la población estudiada $(n=94)$ 
Del total niños parasitados, el $75 \%$ estaba monoparasitado, en tanto que el $25 \%$ estaban infestados con más de un parásito y las combinaciones más frecuentes fueron las siguientes: Blastocys tishominis y Giardia lamblia $(35,3 \%)$; B. hominis y Enterobius vermicularis $(23,5 \%)$ y $\mathrm{B}$. hominis y Entamoeba coli $(17,6 \%)$.

\section{DISCUSIÓN}

El desarrollo de la infancia entre los 6 y 11 años de edad es clave para consolidar las capacidades físicas e intelectuales, la socialización con las demás personas y para formar la identidad y la autoestima. Un niño enfermo disminuye su calidad de vida, sus actividades diarias, su desempeño escolar, sus capacidades físicas y cognitivas $\left({ }^{19)}\right.$. Por otra parte la malnutrición, en cualquiera de sus formas, por exceso o por defecto presenta riesgos considerables para la salud humana ${ }^{(2)}$. La frecuencia de desnutrición encontrada en este trabajo según indicador IMC/edad cercana al 3,9\% es similar al reportado en un trabajo en escolares y adolescentes realizados en7 departamentos del país y a otro realizado en una comunidad rural del departamento de Paraguarí( ${ }^{(4,20)}$. En cambio en otros países como Bolivia, Miranda y cols. reportan en escolares una desnutrición del $20 \%$ y Mamani- Ortiz y cols. cifras de $31,1 \%^{(21,22)}$.

Las enfermedades infecciosas desatendidas son un conjunto de infecciones (muchas de ellas parasitarias), causadas por microorganismos patógenos que afectan poblaciones que viven en condiciones socioeconómicas de pobreza y que tienen problemas de acceso a los servicios de salud ${ }^{(19)}$. La población rural del país no está exenta de esta situación donde encontramos una elevada presencia de parasitosis reflejadas en trabajos nacionales realizados en niños ${ }^{(12,14)}$. La elevada frecuencia de parasitosis hallada en este trabajo, refleja una alta predisposición a las enteroparasitosis, probablemente debidas a deficiente higiene ambiental, y precarias condiciones socioeconómicas en la población estudiada. Esta frecuencia total hallada es inferior a la reportada en niños escolares del primer ciclo de Ciudad del Este $(94,23 \%)$ y superior a lo encontrado en niños escolares de un distrito de Mingá Guazú, Alto Paraná (53\%), así como también en México en un trabajo publicado sobre parasitosis en comunidades rurales donde se encontró una prevalencia de $31,2 \%{ }^{(13,23,24)}$.

El Blastocystis hominis sigue siendo el parásito intestinal más frecuente observado en la población estudiada, al igual que en un trabajo publicado en el país por Pistilli y colaboradores en el año $1997^{(12)}$. Le siguen los demás protozoarios (Giardia lamblia, y Entamoeba coli) que son superiores al de los helmintos y estos resultados coinciden con las frecuencias reportadas en otras publicaciones nacionales ${ }^{(13,14)}$. Según la Organización Mundial de la Salud (OMS) la giardiasis es una enfermedad frecuente y un problema de salud pública, siendo la Giardia lamblia el agente causal, es el protozoo que con más frecuencia causa infección al tubo digestivo del hombre ${ }^{(25)}$. Igualmente en Bolivia se encontró que el $28 \%$ estaban parasitados por este mismo protozoo ${ }^{(26)}$. En relación al total de los parásitos encontrados el $23,5 \%$ eran helmintos coincidiendo con trabajo realizado en México, con una frecuencia encontrada de $28,4 \%{ }^{(27)}$.

La anemia es un deterioro de la salud y un problema a nivel mundial. Ésta afecta a millones de personas en todo el mundo y la máxima prevalencia se da en los niños en edad preescolar, y escolar como refiere un reporte de la $\mathrm{OMS}^{(28)}$. En este trabajo la anemia encontrada fue mayor que la de un estudio realizado en niños en una comunidad rural en el Dpto. de Paraguarí donde fue de $19.1 \%{ }^{(4)}$. Esta frecuencia de anemia hallada es estimada como moderada para la salud pública según la clasificación para la OMS ${ }^{(28)}$. En otros países también se publicaron frecuencias inferiores, así en Venezuela, se encontró un $25,9 \%$ de anemia en niños pre escolares, otro trabajo similar en el mismo país una frecuencia de $16,2 \%$ de anemia ${ }^{(29,30)}$ y en Ecuador una población de escolares reportó una frecuencia de $16,6 \%{ }^{(31)}$.

A pesar de la alta frecuencia de niños que presentaron anemia no se puede saber su etiología debido a que la misma puede deberse a otras causas además de la nutricional o de la parasitosis. El niño con anemia, no necesariamente va a estar bajo de peso; puede estar anémico y tener un peso adecuado. Eso se ve reflejado en el presente trabajo debido a que el $71,8 \%$ de anémicos presentó peso adecuado.

El presente trabajo reveló datos importantes de frecuencia de anemia y parasitosis en población rural en edad escolar por lo que lleva a la necesidad de implementar programas 
para prevenir y detectar oportunamente las mismas evitando así las potenciales complicaciones en la salud de la población infantil.

\section{REFERENCIAS BIBLIOGRÁFICAS}

1. Organización mundial de la salud Nutrición. Disponible http://www.who.int/topics/nutrition/es/ [citado 12 de julio de 2017]

2. Organización mundial de la salud. El departamento de nutrición. OMS. [citado 12 jul. 17]disponible en: http://www.who.int/nutrition/about_us/es

3. FAO. Panorama de la alimentación escolar y posibilidades de compra directa de la agricultura familiar en países de américa latina estudio de caso de Paraguay, agosto de 2013. Disponible en: http://www.fao.org/3/aas834s.pdf

4. Achon F, Cabral L, Vire F, Cabral P, Zavala B. Prevalencia de anemia en la población pediátrica de una comunidad rural del Paraguay y su asociación con el estado nutricional. Rev. ANACEM 2013; 7(1): 7-11.

5. De Benoist B. Worldwide prevalence of anaemia 1993-2005. Base de datos mundial sobre la anemia de la OMS, Ginebra: Organización Mundial de la Salud, Sistema de Información Nutricional sobre Vitaminas y Minerales (VMNIS), 2008.

6. UNICEF, OPS. Panamá. Situación de deficiencia de hierro y anemia [Internet].Panamá: UNICEF; 2006. [citado 12 jul 2017]. Disponible en: http://www.unicef.org/panama/spanish/Hierro .pdf

7. López-Rodríguez MJ, Pérez López MD. Parasitosis intestinales. An Pediatr Contin 2011; 9(4): 249-58. [citado 13 jul. 17] disponible:

pswl.elsevier.es/watermark/ctl_servlet

8. Pistilli N, Zavala de Melgarejo V, Ramirez A, Laviosa de Galeano, Sosa L. Anemia en escolares de zonas aledañas al rio Paraguay en Asunción. Annual Reports. 1998: 51-8.

9. Sandoval NJ. Parasitosis intestinal en países en desarrollo. Rev Med Hondur, 2012; 80(3):89.

10. Carmona-Fonseca J, Correa-Botero A. Parasitosis y desnutrición en niños de Urabá (Colombia) interpretados según las condiciones de vida del país: soledad y olvido. Rev salud ambient. 2013; 13 (2): 108-9.

11. UNICEF Paraguay. Supervivencia y Desarrollo infantil. Situación de la infancia en Paraguay. Disponible en: https://www.unicef.org/paraguay/spanish/sur vival_development_2979.html

12. Pistilli N, Zavala de Melgarejo V, Ramírez A, Laviosa de Galeano, Sosa L. Parasitosis intestinal y anemia en escolares de zonas aledañas al rio Paraguay en Asunción. Annual Reports 1997: 27-32.

13. Cardozo Ocampos GE, Cañete Duarte Z, Lenartovicz V. Frecuencia de enteroparásitos en niños y niñas del primer ciclo de la educación básica de escuelas públicas de Ciudad del Este, Paraguay. Mem Inst Investig Cienc Salud 2015; 13(1): 24-30.

14. Hellman V, Arbo A. Prevalencia de enteroparasitosis en niños de una comunidad Ache de Alto Parará. Rev Inst Med Trop 2016; 11(1):3-9.

15. Tabla de IMC para niños(as) y adolescentes de 5 a 18 años de edad (145-175 cm de estatura) Disponible en: https://www.fantaproject.org/sites/default/file s/resources/FANTA-BMI-charts-Enero2013ESPANOL_0.pdf https://cuidadoinfantil.com/tabla-de-imcpara-ninos-de-5-a-19-anos.html

16. Angel G. Interpretación clínica del laboratorio. 4ta. ed. Buenos Aires: Panamericana; 1993.

17. Campuzano-Maya G. Del hemograma manual al hemograma de cuarta generación. Medicina \& Laboratorio [Internet] 2007 [citado 12 jul 2017]; 13: 511-50. Disponible en:

http://www.medigraphic.com/pdfs/medlab/ myl-2007/myl011-12b.pdf

18. World Health Organization. Basic laboratory in methods medical parasitology. Geneva: 1991. [citado 19 julio 2017]. Disponible en:http://apps.who.int/iris/bitstream/10665 /40793/1/9241544104_\%28part1\%29.pdf

19. Zárate $A$, Ríos $L$, Villalobos $P$. Las parasitosis intestinales asociadas a la pobreza, afectan la calidad de vida y aprendizaje de niños en edad escolar. Revista: $21^{\circ}$ Encuentro Nacional sobre Desarrollo Regional de México18 noviembre 2016. AMECIDER ITM.

20. Morinigo Isla G, Sánchez Bernal S, Sispanov Pankow V, Brizuela Rivarola M, Rolón Villalba G, Mendoza de Arbo L. Perfil nutricional de Escolares y Adolescentes en escuelas públicas y privadas, 2013. Pediatr (Asunción), 2015;42(2): 129-33.

21. Miranda $M$, Olivares $M$, Durán-Pérez J, Pizarro F. Prevalencia de anemia y estado nutricional de escolares del área periurbana de Sucre, Bolivia. Rev Chil Nutr 2015; 42(4): 324-7.

22. Mamani-Ortiz $\mathrm{Y}$, Rojas-Salazar E, CaeroSuarez R, Choque-Ontiveros MC. Prevalencia de desnutrición en niños y niñas en edad escolar del municipio de Vinto. Revista Médico-Científica "Luz y Vida", 2013;4(1): 36-40.

23. Cardozo G, Samudio M. Factores predisponentes y consecuencias de la parasitosis intestinal en escolares paraguayos. Pediatr (Asunción) 2017; 44(2): 117-25.

24. Sánchez de la Barquera-Ramos A, Miramontes-Zapata M. Parasitosis intestinales 
en 14 comunidades rurales del altiplano de México. Rev Mex Patol Clin 2011;5(1):16-25.

25. Almiral $P$, Escobedo A. Enfrentando la giardasis con una nueva mirada. Revista Panamericana de Infectología 2010; 12 (3): $\mathrm{sp}$

26. Amurrio Melgarejo EY, Cuellar JD. Relación del grado de anemia con parasitosis intestinal en niños de 5-14 años en la unidad educativa "Monte Verde"-Provincia Warnes (Junio a diciembre 2013) .UCEBOL 2014;7-12.

27. Gutiérrez-Rodríguez $C$, Trujillo-Hernández $B$, Martínez-Contreras A, Pineda-Lucatero A, Millán-Guerrero R. Frecuencia de helmintiasis intestinal y su asociación con deficiencia de hierro y desnutrición en niños de la región occidente de México. Gac Méd Méx; 2007: 143(4):297-300.

28. Organización Mundial de la Salud. Concentraciones de hemoglobina para diagnosticar la anemia y evaluar su gravedad.
Ginebra: Organización Mundial de la Salud, 2011(WHO/NMH/NHD/MNM/11.1) [citado el 23 de noviembre de 2017]. Disponible en: http://www.who.int/vmnis/indicators/haemogl obin_es.pdf

29. Del Real S, Sánchez A, Barón MA, Díaz N, Solano $L$, Velásquez $E$, et al. Estado Nutricional en niños preescolares que asisten a un jardín de infancia público en Valencia, Venezuela. Archivos Latinoamericanos de Nutrición. 2007:57(3).

30. Barón MA, Liseti $R$, Páez María C, Pabón M. Estado nutricional de hierro y parasitosis intestinal en niños de Valencia, estado Carabobo Venezuela. Anales Venezolanos de Nutrición 2007; 20(1):5-11.

31. Quizhpe E, San Sebastián M, Hurtig AK, Llamas A. Prevalencia de anemia en escolares de la zona amazónica de Ecuador. Rev Panam Salud Pública, 2003; 13 (6): 355-61. 